\title{
High precision beam momentum determination in a synchrotron using a spin-resonance method
}

\author{
P. Goslawski, ${ }^{1, *}$ A. Khoukaz, ${ }^{1}$ R. Gebel, ${ }^{2}$ M. Hartmann, ${ }^{2}$ A. Kacharava, ${ }^{2}$ A. Lehrach,${ }^{2}$ B. Lorentz, ${ }^{2}$ R. Maier,${ }^{2}$ M. Mielke, ${ }^{1}$ \\ M. Papenbrock, ${ }^{1}$ D. Prasuhn, ${ }^{2}$ R. Stassen, ${ }^{2}$ H. J. Stein, ${ }^{2}$ H. Stockhorst, ${ }^{2}$ H. Ströher, ${ }^{2}$ and C. Wilkin ${ }^{3}$ \\ ${ }^{1}$ Institut für Kernphysik, Universität Münster, D-48149 Münster, Germany \\ ${ }^{2}$ Institut für Kernphysik and Jülich Centre for Hadron Physics, Forschungszentrum Jülich, D-52425 Jülich, Germany \\ ${ }^{3}$ Physics and Astronomy Department, UCL, London WC1E 6BT, United Kingdom
}

(Received 21 August 2009; published 23 February 2010)

\begin{abstract}
In order to measure the mass of the $\eta$ meson with high accuracy using the $d p \rightarrow{ }^{3} \mathrm{He} \eta$ reaction, the momentum of the circulating deuteron beam in the Cooler Synchrotron COSY of the Forschungszentrum Jülich has to be determined with unprecedented precision. This has been achieved by studying the spin dynamics of the polarized deuteron beam. By depolarizing the beam through the use of an artificially induced spin resonance, it was possible to evaluate its momentum $p$ with a precision of $\Delta p / p<10^{-4}$ for a momentum of roughly $3 \mathrm{GeV} / c$. Different possible sources of error in the application of the spinresonance method are discussed in detail and its possible use during a standard experiment is considered.
\end{abstract}

DOI: 10.1103/PhysRevSTAB.13.022803

PACS numbers: 29.27.Bd, 29.27.Hj

\section{INTRODUCTION}

For numerous high precision experiments, knowing the beam momentum in an accelerator with the greatest accuracy is essential. Obvious examples of this are investigations of production reactions very close to the thresholds as well as particle mass determinations on the basis of reaction kinematics. Here we present a technique that allows one to determine the momentum of a deuteron beam which is suitable for use in a precise measurement of the mass of the $\eta$ meson.

Measurements of the mass of the $\eta$ meson performed at different experimental facilities over the past decade have resulted in very precise results which differ by up to $0.5 \mathrm{MeV} / c^{2}$, i.e., by more than eight standard deviations. The experiments that are no longer considered in the PDG tables [1] generally involve the identification of the $\eta$ as a missing-mass peak produced in a hadronic reaction. In order to see whether this is an intrinsic problem, and to clarify the situation more generally, a refined measurement of the $d p \rightarrow{ }^{3} \mathrm{He} \eta$ reaction was proposed [2] at the Cooler Synchrotron COSY of the Forschungszentrum Jülich [3].

After producing the $\eta$ mesons through the $d p \rightarrow{ }^{3} \mathrm{He} \eta$ reaction using a hydrogen cluster-jet target [4], the ${ }^{3} \mathrm{He}$ would be detected with the ANKE magnetic spectrometer [5] that is located at an internal-target position of the storage ring. Provided that the reaction is cleanly isolated, the $\eta$ mass can be extracted from pure kinematics through the determination of the production threshold. This requires one both to identify the threshold and to measure accurately the associated beam momentum.

We have previously proved that ANKE has essentially $100 \%$ acceptance for the $d p \rightarrow{ }^{3} \mathrm{He} \eta$ reaction for excess energies $Q$ below about $10 \mathrm{MeV}$ [6], though in that ex-

*paul.goslawski@uni-muenster.de periment the deuteron beam was continuously ramped from below the threshold up to $Q \approx 11 \mathrm{MeV}$. However, although the threshold was well identified, the corresponding value of the beam momentum was only known in the experiment with a relative accuracy of about $10^{-3}$.

For the new $\eta$ mass proposal [2], the decision was taken to measure at 13 fixed energies in the range $1<Q<$ $10 \mathrm{MeV}$ as well as $Q=-5 \mathrm{MeV}$ for background studies. To determine the mass using this kinematic method with a precision that is competitive with other recent measurements, i.e., $\Delta m_{\eta}<50 \mathrm{keV} / c^{2}$ [1], the associated beam momenta have to be fixed with an accuracy of $\Delta p / p<$ $10^{-4}$. This requires the 13 beam momenta in the range of $3100-3200 \mathrm{MeV} / c$ to be measured to better than $300 \mathrm{keV} / c$.

Generally at synchrotron facilities like COSY, the velocity of the beam particles, and hence the beam momentum, is determined from the knowledge of the revolution frequency combined with the absolute orbit length. The accuracy that can be reached using this technique is limited by the measurement of the orbit length by, e.g., beam position monitors. This is in the region of $\Delta p / p \approx 10^{-3}$ and so an order of magnitude improvement is needed for the $\eta$ mass experiment. Because of the technical limitations of such a macroscopic device, it is not feasible to obtain the necessary increase in accuracy by simply scaling up the number of beam pickup electrodes. The beam momentum must therefore be determined in some other way.

The method proposed for electron colliders more than 30 years ago to overcome this problem $[7,8]$ has been very successfully applied at the VEPP accelerator of the BINP at Novosibirsk to measure the masses of a wide variety of mesons from the $\phi$ to the $Y$ [9]. The technique was further developed at DORIS in Hamburg [10] and CESR in Cornell [11] as well as LEP at CERN [12]. 
The spin of a polarized beam particle precesses around the normal to the plane of the machine, which is generally horizontal. The spin can be perturbed by the application of a horizontal rf magnetic field from, for example, a solenoid. The beam depolarizes when the frequency of the externally applied field coincides with that of the spin precession in the ring. The usefulness of the technique relies on the fact that a frequency $f$ can be routinely measured with a relative precision of $\Delta f / f=10^{-5}$. Furthermore, the position of the depolarizing resonance depends purely upon the revolution frequency of the machine and the kinematical factor $\gamma=E / m c^{2}$, where $E$ and $m$ are the particle total energy and mass, respectively. The measurements of the revolution and depolarizing frequencies together allow the evaluation of $\gamma$ and hence $E$ and the beam momentum $p$.

There is no in-principle reason why the induceddepolarization approach should not be equally applicable to other beam particles with an intrinsic spin, such as protons or deuterons. In fact, the effects have recently been confirmed at COSY in studies of the spin manipulation of both polarized proton [13] and deuteron beams [14]. This is the methodology that we are pursuing at COSY for the measurement of the $\eta$ mass. For the first time in 2007 it was possible in a test run to reach an accuracy in the beam momentum calibration of $\Delta p / p<10^{-4}$ using the technique with a coasting beam but no internal target [15]. In the present paper we describe how the method can be used in a standard beam time under normal experimental conditions in the presence of a thick internal target.

In Sec. II we describe the physical principles underlying the spin-resonance method. After discussing the behavior of a vector polarized deuteron beam in COSY, we show how to induce an artificial spin resonance to depolarize the beam. The experimental conditions that allow one to determine the two critical observables are explained in Sec. III. The revolution frequency $f_{0}$ is measured via the Schottky noise of the beam and the spin-resonance frequency $f_{r}$ using the rf solenoid and the EDDA detector as a beam polarimeter [16]. The deuteron beam results are presented in Sec. IV, where the estimated uncertainties are discussed in some detail. Our conclusions are summarized in Sec. V.

\section{THEORETICAL BACKGROUND OF THE SPIN-RESONANCE METHOD}

\section{A. Spin in synchrotrons}

In contrast to the case of a spin-half fermion such as an electron or proton, the deuteron is a spin-one boson that can be placed in three magnetic substates $m=-1,0+1$, and the resulting polarization phenomenology is more complex. Eight independent parameters are necessary to characterize a spin-one beam, three for the vector polarization and five for the tensor [17]. However, only the vector polarization,

$$
P_{V}=\left(N_{+}-N_{-}\right) / N
$$

is used in the present experiment for the spin-resonance method since it can be measured with the beam polarimeter to a higher precision than the tensor. Here $N_{m}$ is the number of particles in state $m$ and $N=N_{+}+N_{-}+N_{0}$ is the total number of particles.

The motion of the spin vector $\vec{S}$, defined in the rest frame of the particle, in a circular accelerator, synchrotron, or storage ring, is given by the Thomas-Bargmann-MichelTelegdi equation [18]:

$$
\begin{aligned}
\frac{d \vec{S}}{d t}= & \frac{e}{\gamma m} \vec{S} \times\left[(1+\gamma G) \vec{B}_{\perp}+(1+G) \vec{B}_{\|}\right. \\
& \left.+\left(G \gamma+\frac{\gamma}{\gamma+1}\right) \frac{\vec{E} \times \vec{\beta}}{c}\right]
\end{aligned}
$$

where $\vec{B}_{\perp}$ and $\vec{B}_{\|}$are the transverse and longitudinal components of the magnetic fields of the accelerator in the laboratory frame and $\vec{E}$ represents the electric field. The velocity of the particle is $\vec{\beta} c$, in terms of which $\gamma=$ $1 / \sqrt{1-\beta^{2}}$.

In a synchrotron without horizontal magnetic fields and where the electric field is always parallel to the particle motion, the spin motion only depends on the first term, i.e., is a function of the transverse magnetic fields $\vec{B}_{\perp}$ of the accelerator. The deuteron spin precesses around the stable spin direction, which is given by the vertical fields of the guiding dipole magnets of the synchrotron. The number of spin precessions during a single circuit of the machine, the spin tune $\nu_{s}$, is proportional to the particle energy. In the coordinate basis of the moving particle, the spin tune is given by

$$
\nu_{s}=G \gamma,
$$

whereas, taking into account the extra rotation associated with a single circuit of the machine, this becomes $\nu_{s}=$ $1+G \gamma$ in the laboratory frame. Here $G=(g-2) / 2$ is the gyromagnetic anomaly of the particle, where $g$ is the gyromagnetic factor. For deuterons the gyromagnetic anomaly, $G_{d}=-0.1429872725 \pm 0.0000000073$, can be calculated from the ratios of the magnetic moments and masses of the proton and deuteron [19].

\section{B. Artificially induced depolarizing resonances}

The beam polarization can be perturbed by a horizontal magnetic field in the synchrotron and, if the frequency of the perturbation coincides with the spin-precession frequency, the beam depolarizes. One kind of first-order resonance is the imperfection resonance. If the spin tune is an integer, then the horizontal imperfection fields of the synchrotron can interact resonantly with the particle spin, building up effects coherently turn by turn. The positions in momentum of the depolarizing resonances depend on the gyromagnetic anomaly of the particle. In contrast to the 
case of protons, where the first imperfection resonance occurs at a momentum of $464 \mathrm{MeV} / c$, the first for deuterons is at $13 \mathrm{GeV} / c$, which is well outside the COSY momentum range. Furthermore, in the present experiment the spin tune remains in the region of $\nu_{s}=0.2775-0.2818$.

Because of the betatron oscillation frequency of the circulating beam, the particles can also encounter the fields of the focusing quadrupole magnets in resonance with the spin tune, which lead to a second kind of first-order spin resonance, the so-called intrinsic resonance. These resonances also occur only for energies that are far beyond the COSY deuteron momentum and working point range [21].

A horizontal rf field from a solenoid can lead to rfinduced depolarizing resonances. Depending on the form of the field, these can be used to depolarize the beam, to measure the spin tune, or even to flip the spin direction of the beam particles. The spin-resonance frequency for a planar accelerator where there are no horizontal fields is given by [8]

$$
f_{r}=(k+\gamma G) f_{0}
$$

where $f_{0}$ is the revolution frequency of the beam, $\gamma G$ is the spin tune, and $k$ is an integer. If the rf frequency of the perturbation is close to $f_{r}$ then the polarization of the beam is maximally influenced. Horizontal magnetic fields in the accelerator lead to modifications of Eqs. (3) and (4) $[12,22]$. To avoid this complication, all solenoidal and toroidal magnets in the COSY ring, those of the experiment as well as those of the electron cooler, were switched off. Residual shifts in the resonance frequency arising from field errors and vertical orbit distortions were estimated and found to be negligibly small. These effects are discussed in more detail in Sec. IV C.

It is important to note that Eq. (4) is only valid if, as is the case for the present experiment, there is no full or partial Siberian snake. The resonance with $k=1$ was used as this matches the frequency range of the rf solenoid installed at COSY. The kinematic $\gamma$ factor, and thus the beam momentum, can be determined purely by measuring both the revolution and spin-resonance frequencies.

\section{EXPERIMENTAL CONDITIONS}

The COSY accelerator facility is presented in Fig. 1. After preacceleration in the cyclotron JULIC, COSY can provide unpolarized and polarized proton and deuteron beams in the momentum range of 300-3700 MeV/c. For the present experiment, two of the four internal facilities were used, viz. ANKE with a thick hydrogen cluster-jet target and EDDA [16] as beam polarimeter [23]. The beam was accelerated with the rf cavity and the barrier bucket (bb) cavity was used to compensate for the energy losses incurred through the beam-target interactions (see Sec. III A). The position of the rf solenoid is also shown. The integrated value of the solenoid's maximum longitu-

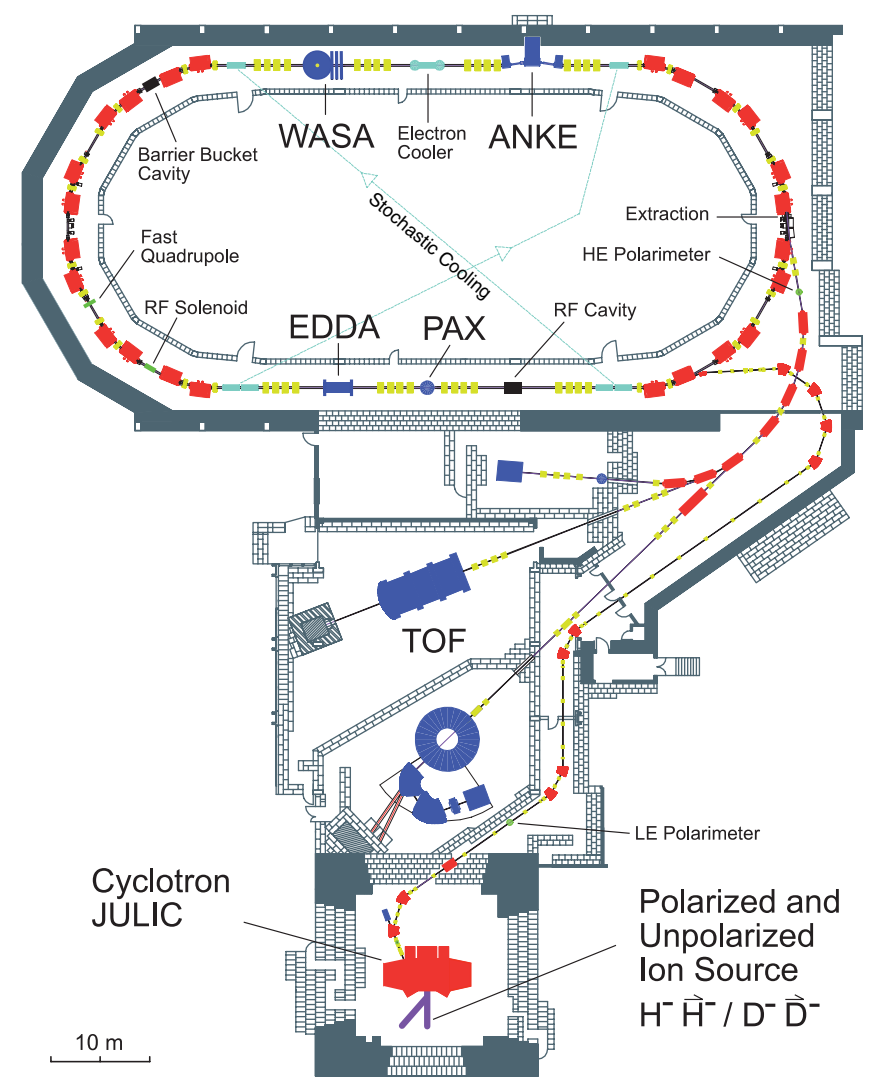

FIG. 1. (Color) The COSY accelerator facility. The cyclotron JULIC provides both unpolarized and polarized proton and deuteron beams for injection into the COSY ring, where they are accelerated and stored. COSY operates in the momentum range of 300-3700 MeV/c. The position of the ANKE spectrometer with the thick internal hydrogen cluster-jet target is shown, as are those of the rf solenoid to depolarize the deuteron beam, the barrier bucket cavity to compensate beam-target energy losses, and the EDDA detector that was used as a beam polarimeter.

dinal rf magnetic field is $\int B_{\mathrm{rms}} d l=0.67 \mathrm{Tmm}$ at a $\mathrm{rf}$ voltage of $5.7 \mathrm{kV} \mathrm{rms}$. Its frequency range is $0.5-1.5 \mathrm{MHz}$.

\section{A. The rf cavity system}

For a high precision experiment it is crucial that the beam momentum remains stable throughout the whole accelerator cycle. In a typical cycle of a standard scattering experiment at ANKE, the beam is first injected into COSY and accelerated to the nominal momentum. The rf cavity is then switched off to provide a coasting beam that fills the ring uniformly. This then gives constant count rates, which reduces the dead time of the data acquisition system (DAQ). But, because of the energy losses of the charged beam particles through electromagnetic processes as the beam passes repeatedly through the target, the momentum changes and this leads to a shift in the revolution frequency [24]. For a deuteron beam and a hydrogen cluster-jet target with a density of $\rho=1 \times 10^{15} \mathrm{~cm}^{-2}$, the revolution fre- 
quency would change by up to $103 \mathrm{~Hz}$ over a $180 \mathrm{~s}$ long cycle, corresponding to a shift in beam momentum of 2. $2 \mathrm{MeV} / c$.

To compensate for this effect and to guarantee a constant beam momentum over the whole data-taking cycle, a second cavity, the barrier bucket (bb) cavity [25], was switched on after the rf cavity was switched off. In this way a beam with a constant momentum over the whole cycle could be produced that filled roughly $80 \%-90 \%$ of the ring homogenously and thus achieved the necessary reduction in the dead time of the DAQ.

\section{B. The cycle timing and the supercycle}

The 13 closely spaced energies studied near the $\eta$ threshold were divided into two so-called supercycles that involved up to eight different COSY machine settings. The first and the second supercycle each consisted of seven different energies where, to allow comparison between the two sets, the first energies of the two supercycles were chosen to be identical. Data at 13 different energies were therefore recorded. The different machine settings in the supercycles were imposed sequentially, after which the supercycle was repeated. Each supercycle was used for five days of continuous Schottky data taking to study the long term stability of COSY and to take data in parallel for the $\eta$ meson mass determination. The reason for choosing supercycles instead of independent measurements at fixed energies was to guarantee the same experimental conditions for each of the beam energies in one supercycle. In this way the systematic uncertainties could be investigated in more detail, as will be discussed in Sec. IV.

Before starting each of the five day blocks, the individual beam energies were measured using $36 \mathrm{~s}$ accelerator cycle lengths. The timing structure of the accelerator cycles is described in Table I. After the injection of the beam into COSY, the stored deuterons were accelerated to the first nominal beam energy of the supercycle using the regular COSY rf cavity. At $t=3.7 \mathrm{~s}$ this cavity was switched off and at $t=4 \mathrm{~s}$ the bb cavity was brought into operation to compensate for the beam energy losses. At $t=20 \mathrm{~s}$ the amplitude of the depolarizing rf solenoid was linearly ramped from 0 to $2.4 \mathrm{kV}$ rms to produce a $\int B_{\mathrm{rms}} d l=0.29 \mathrm{~T} \mathrm{~mm}$ in $200 \mathrm{~ms}$, remained constant for

TABLE I. Cycle timings used to determine the spin-resonance frequency spectrum with the polarized beam.

\begin{tabular}{lc}
\hline \hline Time (s) & Process \\
\hline 0 & Start of cycle: injection \\
$0-3.7$ & Acceleration of the beam with rf cavity \\
3.7 & Switch off rf cavity \\
4 & Switch on bb cavity \\
$20-25$ & rf solenoid on \\
$25-30$ & Polarization measurement with EDDA \\
36 & End of cycle \\
\hline \hline
\end{tabular}

$5 \mathrm{~s}$, and was then ramped down in $200 \mathrm{~ms}$. This was followed by a beam polarization measurement for five seconds using the EDDA detector [16]. At $t=36 \mathrm{~s}$ the cycle was terminated. This procedure was repeated at the same beam energy but with different rf solenoid frequencies in order to obtain the spin-resonance spectrum. After completion of this first submeasurement, the next beam energy of the supercycle was used and the corresponding spin-resonance spectrum measured until complete data was obtained at all the energies of the supercycle.

After measuring the spin-resonance spectrum, the supercycle was switched on for five days of continuous data taking to investigate the long term stability of the COSY accelerator. For this study the polarization measurements were omitted and total cycle lengths of $206 \mathrm{~s}$ were used. After injection, acceleration, and starting the bb cavity, Schottky measurements were performed over the time interval of $t=14-196 \mathrm{~s}$. The eight beam energies in one supercycle (the first one is installed twice) involved a total time of $1648 \mathrm{~s}$, after which the supercycle was repeated. After the five days of data taking, the system was returned to the conditions of Table I to repeat the measurement of the spin-resonance spectrum in order to control systematic effects.

The polarized ion source at the injector cyclotron of COSY currently gives a beam intensity that is about an order of magnitude too low compared to that which is required for the $\eta$ mass proposal. It was therefore decided to use this ion source only for the beam energy measurement before and after the supercycles. As a consequence, for the long term stability studies COSY was switched to the unpolarized ion source, which allowed beam intensities up to $n_{d} \approx 1 \times 10^{10}$. However, it had to be carefully checked that the same COSY beam energies were obtained when using the polarized and the unpolarized ion sources. To ensure this, the complete settings of the cyclotron, the beam injection, as well as COSY itself, were fixed when switching from one ion source to the other. The revolution frequencies $f_{0}$ of the stored beam in the two cases matched to within $\Delta f_{0} \leq 6 \mathrm{~Hz}$, proving the validity of this method. The determination of $\Delta f_{0}$ was limited in the present case by the experimental resolution of the Schottky spectrum analyzer, though this could be improved by better calibration.

Both in the beam energy determination, as well as later in the Schottky data-taking time, one had to be assured that the measurements within the cycles were started sufficiently long after the ramping of the COSY dipole magnets for the acceleration of the beam. Otherwise, the not-yetstable magnetic fields would lead to deviations in the values determined for the beam momentum. Detailed measurements of the beam energy by the spin-resonance method as a function of the time in the cycle showed that the experimental situation is already stable ten seconds after the start of the cycle [26]. Therefore, the rf solenoid 
field and the Schottky data taking were started 20 and $14 \mathrm{~s}$ after injection, respectively. As a further check, measurements showed that the same beam energy was observed close to the end of the cycle as at the beginning [26] (see Sec. IV B). Thus it is valid to investigate the beam energy at one fixed time during the cycle and to take the resulting value as representative for the whole cycle.

\section{Determination of the revolution frequency $f_{0}$ via the Schottky noise measurements}

The revolution frequency $f_{0}$ was measured by using the Schottky noise of the deuteron beam. The origin of this effect is the statistical distribution of the charged particles in the beam. This leads to random current fluctuations that induce a voltage signal at a beam pickup in the ring. The Fourier transform of this voltage-to-time signal by a spectrum analyzer delivers the frequency distribution around the harmonics of the revolution frequency of the beam.

For the measurement of the Schottky noise, the beam pickup and the spectrum analyzer of the stochastic cooling system of COSY were used. The spectrum analyzer (standard swept-type model HP 8595E) is sensitive to the Schottky noise current, which is proportional to the square root of the number $N$ of the particles in the beam. To get the Schottky power spectra, which represent the momentum distribution [27], the amplitudes of the measured distribution were squared. The spectrum analyzer was operated in the range of the thousandth harmonic but, because of the different flattop revolution frequencies, harmonics from 997 to 1004 were also measured.

The Schottky spectra were recorded every $30 \mathrm{~s}$ throughout the whole beam time so that altogether nearly 15000 distributions were collected and sorted by energy, i.e., by

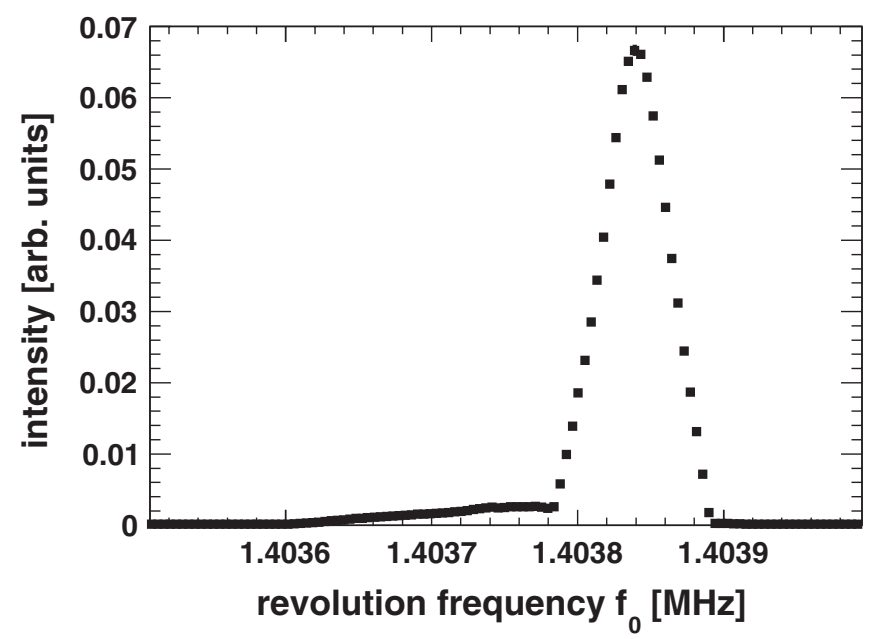

FIG. 2. Mean Schottky power spectrum extracted from measurements over five days at one energy. The statistical error bars lie within the data points. By calculating the weighted arithmetic mean, an average revolution frequency of $\bar{f}_{0}=1403831.75 \pm$ $0.12 \mathrm{~Hz}$ was deduced. flattop. This large number of Schottky measurements allows the study of the long term stability of the revolution frequency, which will be discussed in Sec IV. From all the spectra taken over five days that were measured under the same conditions at a particular energy, one mean spectrum was calculated, an example of which is presented in Fig. 2.

The full width at half maximum is in the region of 40$50 \mathrm{~Hz}$ for all energies. The position of the mean distribution of the circulation frequency is stable for the whole cycle time but, within the cycle, a small tail is seen at lower frequencies. This corresponds to beam particles that escaped the influence of the bb cavity but still circulated in COSY. By calculating the weighted arithmetic mean of the revolution frequency distribution, an average revolution frequency was estimated. The statistical uncertainty of the mean revolution frequency, which is below $0.2 \mathrm{~Hz}$ for all energies, depends on both the number of measured Schottky spectra and on the distribution variations.

\section{Determination of the spin-resonance frequency $f_{r}$ via an induced spin resonance}

For all 13 energies the spin-resonance spectrum was measured twice, once before and once after the five days of Schottky data taking, as described in Sec. III B. The polarization of the beam leads to an asymmetry in scattering from a carbon target, which was measured with the EDDA detector [28]. For our purposes absolute calibrations of this device at the different energies were not required; a quantity merely proportional to the polarization such as the left-right asymmetry is sufficient.

An example of a spin-resonance spectrum at one energy is shown in Fig. 3. This displays the non-normalized polarization ("relative polarization") as a function of the solenoid frequency. Far away from the spin resonance at 1.0116 and $1.0120 \mathrm{MHz}$, a high beam polarization was

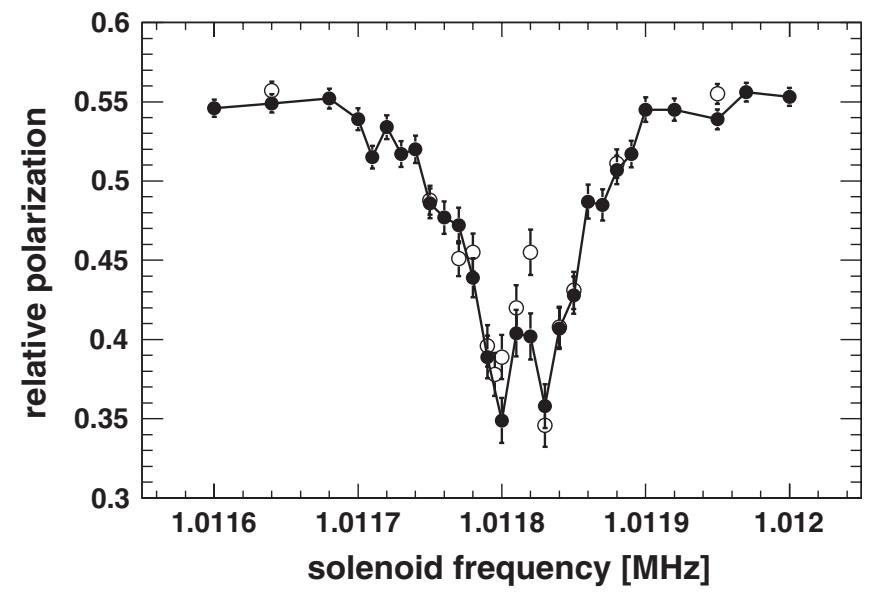

FIG. 3. Spin-resonance measurements at one energy (closed circles). The cycle timings are described in Table I. The open symbols represent results obtained for an extended cycle time, where the perturbing solenoid was switched on after $178 \mathrm{~s}$. 
measured. In contrast, when the frequency of the solenoid coincided with the spin-precession frequency, the beam was maximally depolarized. The full width at half maximum was in the region of $80-100 \mathrm{~Hz}$ for all energies. Unlike the earlier spin-resonance test measurement with a coasting beam, i.e., no cavities and no internal target [15], the spin-resonance spectra are not smooth. The structures, especially the double peak in the center, are caused by the interaction of the deuteron beam with the bb cavity. However, by comparing the spin-resonance spectra measured for an unbunched and bunched beam with accelerating cavity with $h=1$ or the barrier bucket cavity, it was found that the centers of gravity of the spectra were the same.

To study the shapes of the spin-resonance spectra in more detail, all 26 distributions were fitted with Gaussians and then shifted along the abscissa so that the mean value of each individual spectrum was zero. In addition, each spectrum was shifted along the ordinate so that the off-resonance polarization vanished. Finally, the

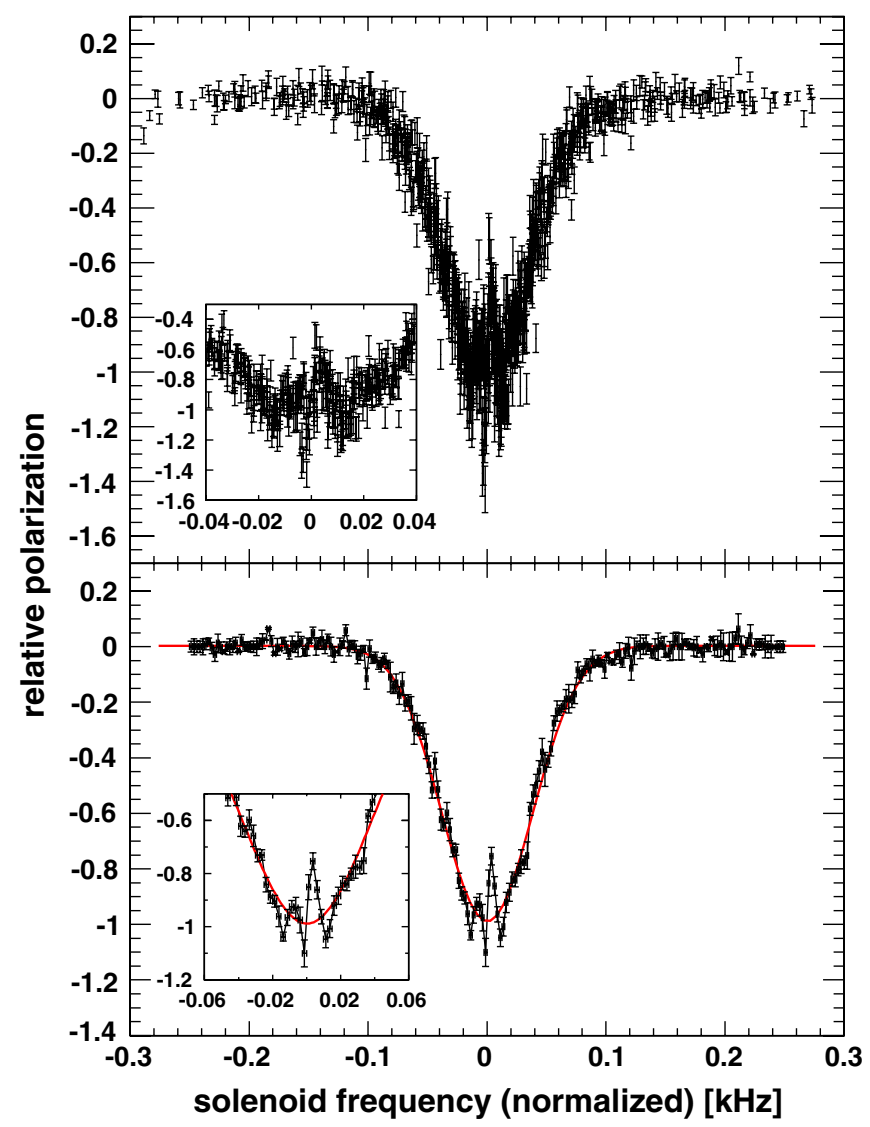

FIG. 4. (Color) Panel (a): The spin-resonance spectra normalized by a Gaussian. Panel (b): The same but with larger bins. The spin-resonance shape is symmetric about zero and smooth except in the center, where a double peak structure is seen. The structures, especially the double peak in the center, are caused by the interaction of the deuteron beam with the bb cavity. The insets show the resonance valley in greater detail. data were scaled to a uniform height and displayed together in a single plot to allow a comparison of all the spectra. The resulting global spin-resonance spectrum shown in Fig. 4(a) is symmetric around zero and smooth, except for the structure at the center. This region is shown in greater detail in the inset. In order to improve the visibility of the structures close to the minimum, the size of the frequency bins was increased and the results displayed in Fig. 4(b).

A structure with a symmetric double peak and an oscillation is observed in the center of the spin resonance. However, it is important to note that the Gaussian mean value, i.e., the spin-resonance frequency, is not influenced by this structure. This was checked by making a fit where the data points at the center were excluded. The spinresonance frequencies $f_{r}$ were extracted from the spinresonance spectra for all energies by making Gaussian fits. These gave $\chi^{2} /$ ndf in the region of $2-3$. The statistical uncertainties of the spin-resonance frequencies are on the order of $1-2 \mathrm{~Hz}$ at $f_{r} \approx 1.01 \mathrm{MHz}$.

\section{RESULTS}

\section{A. Stability of the revolution frequency $f_{0}$}

The bb cavity compensates the effects of beam-target energy losses and should ensure that the revolution frequency remains constant. The large number of Schottky measurements allowed us to study the long term stability and to identify the magnitude of the variations of the revolution frequency at COSY. Therefore all the Schottky spectra at one energy from one day were analyzed and the mean revolution frequency of that day calculated, as described in Sec III C.

In addition, the revolution frequencies for these data were calculated for every four hours to study the daily variation of the circulation frequency. The differences between the revolution frequencies of every four hours and the mean frequency of the day are presented in the upper part of Fig. 5. To study the variation of the revolution frequency over the five days of data taking, the same procedure was carried out for the Schottky data measured over this period. The differences between the mean revolution frequencies of every day and the mean frequency of the whole five days of data taking are presented in the bottom part of Fig. 5. The horizontal bars represent the time intervals for which the revolution frequency was evaluated.

The analysis shows that the revolution frequency at COSY over one day and also over five days is very stable. The variations of the revolution frequency are very small, being on the order of $1 \mathrm{~Hz}$ at a circulation frequency of $f_{0} \approx 1.4 \mathrm{MH} z$. In sum, it was possible to determine the revolution frequencies for all energies with a statistical uncertainty below $1 \mathrm{~Hz}$. Nevertheless the much larger systematic uncertainty of $\Delta f_{0}=6 \mathrm{~Hz}$ dominated the precision, and this arose from the preparation of the Schottky 

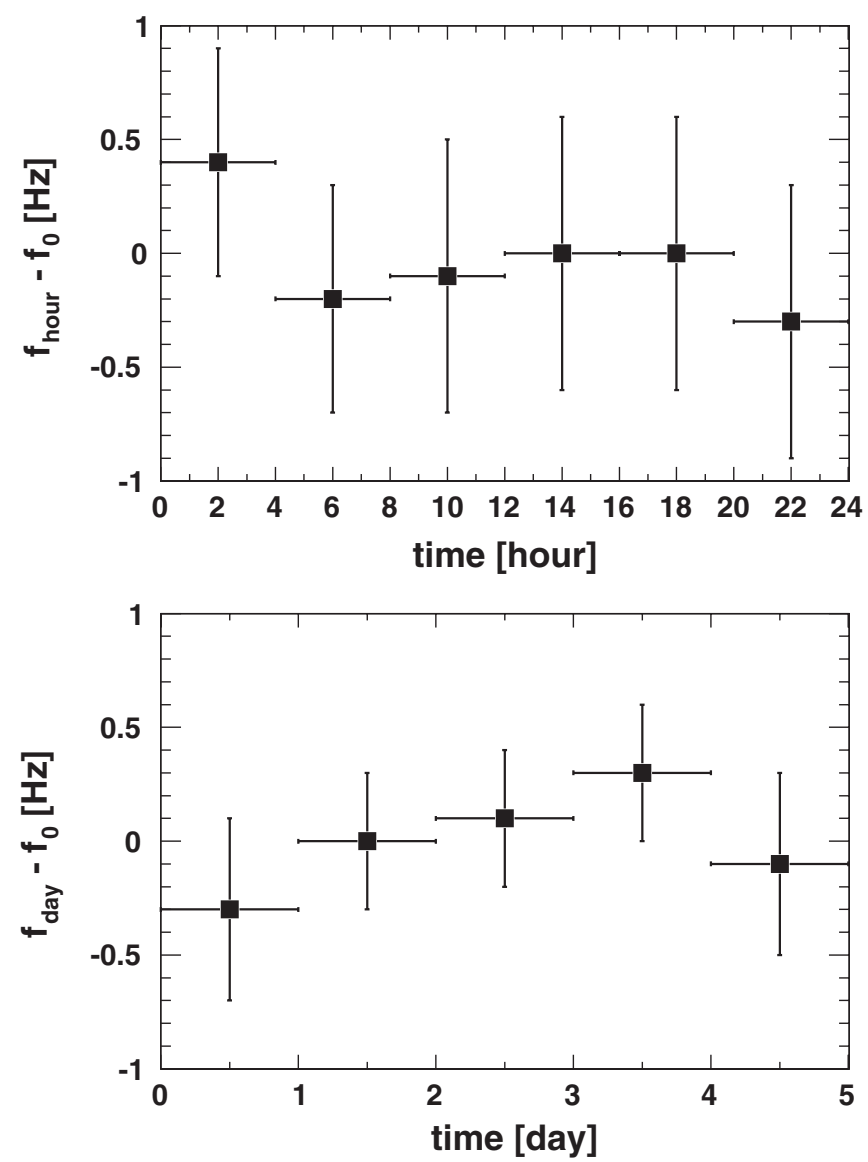

FIG. 5. Stability of the revolution frequency $f_{0}$. In panel (a) the differences between the revolution frequencies for every four hours and the mean revolution frequency of the day are shown. In panel (b) the differences between the revolution frequencies for each day and the mean revolution frequency of the five days of Schottky data taking are shown. From these figures it is clear that the revolution frequency at COSY is very stable, with variations below $1 \mathrm{~Hz}$ at a circulation frequency of $f_{0} \approx 1.4 \mathrm{MHz}$.

spectrum analyzer used. A more refined calibration of this device could improve the systematic precision of the circulation frequency measurement down to $1 \mathrm{~Hz}$.

\section{B. Spin-resonance frequency $f_{r}$}

It is important for the interpretation of the spinresonance measurements to know to what extent the positions of the observed spin-resonance frequencies are stable over the finite accelerator cycle in the presence of a thick internal target. Therefore, in a special measurement, the switch-on of the rf solenoid was delayed from 20 to $178 \mathrm{~s}$ in order to investigate the position of the spin-resonance frequency close to the end of a long cycle. The observed data (open symbols of Fig. 3) showed a resonance position which agreed with the data taken at the beginning of the cycle to within $2 \mathrm{~Hz}$.

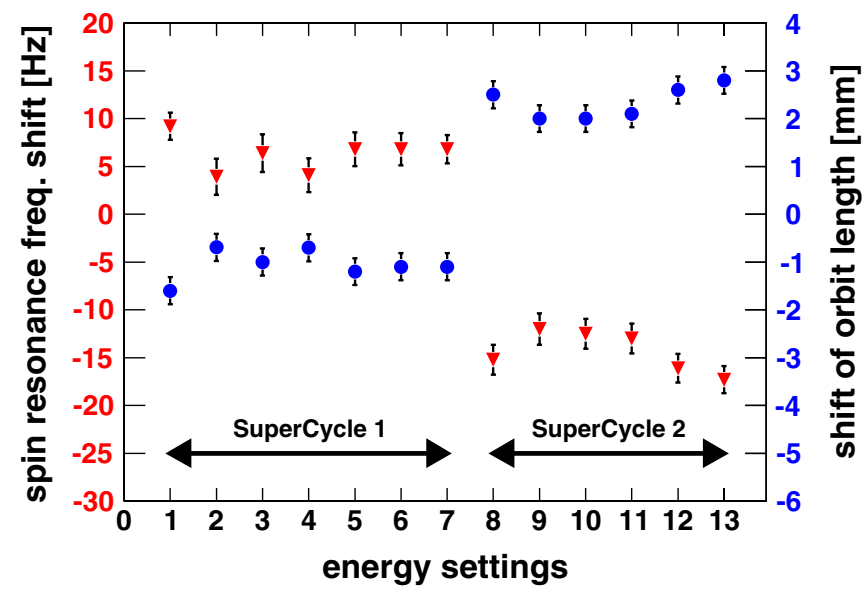

FIG. 6. (Color) The spin-resonance frequencies were measured twice, once before and once after the five days of data taking. The red triangles present the shift of the spin-resonance frequency $f_{r}$ from the first to the second measurement. These shifts correspond to changes in the orbit length, which are shown as blue circles. For the first supercycle, the spin-resonance frequencies decrease between the two measurements by $4-10 \mathrm{~Hz}$, which corresponds to an increase in the orbit length in the range of $0.7-$ $1.6 \mathrm{~mm}$. For the second supercycle an increase of the spin resonance in the range of $12-17 \mathrm{~Hz}$ was observed, i.e., a decrease in the orbit length in the range of $2.0-2.8 \mathrm{~mm}$.

In Fig. 6 the shifts between the first and second spinresonance measurements are shown as red triangles for all 13 energies. The frequencies in the first supercycle decrease by between 4 and $10 \mathrm{~Hz}$ for all energies, whereas for the second supercycle they increase in the range of 12$17 \mathrm{~Hz}$. These systematic shifts of the frequencies in the same direction indicate slight changes in the COSY settings. Because the revolution frequency is stable, as described in Sec. IVA, the change is attributed to a shift in the orbit length $s$.

The velocity $v$ of the particle is the product of the revolution frequency and the orbit length $v=s f_{0}$. Using Eq. (4), the orbit length can be calculated from the revolution and the spin-resonance frequencies:

$$
s=c\left[\frac{1}{f_{0}^{2}}-\left(\frac{G_{d}}{f_{r}-f_{0}}\right)^{2}\right]^{1 / 2},
$$

which allows the orbit lengths to be extracted with a precision better than $0.3 \mathrm{~mm}$ for every flattop. Since the nominal COSY circumference is $183.4 \mathrm{~m}$, this gives a relative accuracy of $\Delta s / s \leq 2 \times 10^{-6}$. The uncertainty is dominated by that of the spin-resonance frequency. The shift in the spin-resonance frequency corresponds to a change in the orbit length of up to $3 \mathrm{~mm}$, which is presented for all energies in Fig. 6 as blue circles. The shifts of the spin-resonance frequencies of the first supercycle suggest an increase in the orbit length in the range of $0.7-$ $1.6 \mathrm{~mm}$ and to a decrease in the range of $2.0-2.8 \mathrm{~mm}$ for the second supercycle. 
To determine the precise beam momenta, the mean value of the two spin-resonance measurements for every energy was calculated. These mean values differ by up to $10 \mathrm{~Hz}$ from the single spin-resonance measurements. Nevertheless, in view of the observed shift of the spin-resonance frequency, a very conservative systematic uncertainty of $\Delta f_{r}=15 \mathrm{~Hz}$ was assumed.

\section{Accuracy and systematic shifts of the resonance frequency}

One obvious limitation on the spin-resonance method is given by the uncertainty in the deuteron gyromagnetic anomaly $G_{d}$. However, this leads to a relative precision in the beam momentum of $\Delta p / p=5 \times 10^{-8}$, which can be safely neglected.

The first-order uncertainties in the momentum measurement depend on the accuracies to which the spin resonance and revolution frequencies are determined. As described in Sec. IVA and IV B, these are $15 \mathrm{~Hz} / 1.01 \mathrm{MHz}=1.5 \times$ $10^{-5}$ and $6.0 \mathrm{~Hz} / 1.40 \mathrm{MHz}=4.3 \times 10^{-6}$, respectively. The error therefore arises primarily from the measurement of the spin-resonance frequency.

The intrinsic width of the spin resonance may also impose a limit on the accuracy achievable. In this experiment, the integrated value of the solenoid's maximum longitudinal $\mathrm{rf}$ magnetic field gives a resonance strength of about $\epsilon=3 \times 10^{-6}$, which leads to a spin resonance with a FWHM width $\approx 9 \mathrm{~Hz}$. This is much smaller than the observed width of $80-100 \mathrm{~Hz}$, which is therefore dominated by the momentum spread of the beam. Higher order contributions lead to an additional spread in the spin frequencies caused by nonlinear synchrotron and betatron motion [29]. It should be stressed that these higher order effects, which are negligible compared to the calculated resonance width, do not contribute to a shift of the resonance frequencies.

Systematic shifts of the resonance frequencies may be caused by deviations from idealized conditions in a real accelerator like COSY. The possible effects and their contribution to the accuracy of the resonance frequency determination were estimated and are summarized in Table II.

Radial and longitudinal fields in the accelerator may lead to a modification of Eq. (4) [22], i.e., to a systematic shift of the resonance frequency. Even though all solenoidal and toroidal fields, which may act as partial Siberian snakes, were turned off for this experiment, field errors and vertical orbit distortions could generate some net radial or longitudinal fields $[9,12]$. These effects were estimated for the current experimental conditions and found to be negligibly small. The typical field errors of the main magnets, $\Delta B / B \approx 2 \times 10^{-4}$, would lead to a shift in the spinresonance frequency of $\Delta f_{r} / f_{r}<1.4 \times 10^{-9}$. Similarly, the observed vertical orbit displacement of $\Delta y_{\text {rms }}<$ $1.8 \mathrm{~mm}$ would induce a shift of $\Delta f_{r} / f_{r}<6.0 \times 10^{-9}$.

The largest contribution to a systematic shift of the resonance frequency could come from the vertical closed orbit deviations in the quadrupole magnets of the ring. However, this contribution of $\Delta f_{r} / f_{r}=4 \times 10^{-8}$ is comparable to the in-principle limitation of the method arising the knowledge of the deuteron $G$ factor. It is over two orders of magnitude below the accuracy achieved in the experiment.

\section{Determination of the deuteron beam momenta $p$ and the momentum smearing $\Delta p / p$}

The deuteron kinematic $\gamma$ factor and the beam momenta were calculated according to Eq. (6):

$$
\gamma=\frac{1}{G_{d}}\left(\frac{f_{r}}{f_{0}}-1\right) \quad p=m_{d} \beta \gamma=m_{d} \sqrt{\gamma^{2}-1}
$$

from the knowledge of the revolution and the spinresonance frequencies. The accuracies to which both frequencies are determined are dominated by systematic effects. The revolution frequency measured by the Schottky spectrum analyzer has an uncertainty of $\Delta f_{0}=6 \mathrm{~Hz}$, corresponding to one in the beam momentum of $50 \mathrm{keV} / c$. The error in the determination of the spin-resonance frequency $\Delta f_{r}=15 \mathrm{~Hz}$ arises from the small variations of the orbit length and corresponds to an uncertainty in the beam momentum of $164 \mathrm{keV} / c$. Because these systematic uncertainties are independent, they are added quadratically to give a total uncertainty $\Delta p / p \leq 6 \times 10^{-5}$, i.e., a precision of $170 \mathrm{keV} / c$ for beam momenta in the range of $3100-3200 \mathrm{MeV} / c$. This is over an order of magnitude better than ever reached before for a standard experiment in the COSY ring. An example of the reconstructed beam properties is presented in Table III for one typical energy setting. The measured beam momentum differed by $\approx 5 \mathrm{MeV} / c$ from the nominal requested momentum.

Two further quantities, the beam momentum smearing $\delta p / p$ and the smearing of the orbit length $\delta s / s$, can be extracted from the spin-resonance spectra. As discussed in

TABLE II. Accuracy and possible systematic shifts of the resonance frequency $f_{r}$.

\begin{tabular}{lc}
\hline \hline Source & $\Delta f_{r} / f_{r}$ \\
\hline Resonance frequency accuracy from depolarization spectra & $1.5 \times 10^{-5}$ \\
Spin tune shifts from longitudinal fields (field errors) & $1.4 \times 10^{-9}$ \\
Spin tune shifts from radial fields (field errors, vertical correctors) & $6.0 \times 10^{-9}$ \\
Spin tune shifts from radial fields (vertical orbit in quadrupoles) & $4.1 \times 10^{-8}$ \\
\hline \hline
\end{tabular}


TABLE III. Typical results for one beam setting.

\begin{tabular}{lc}
\hline \hline Nominal beam momentum & $3150.5[\mathrm{MeV} / c]$ \\
\hline Revolution frequency & $1403832 \pm 6[\mathrm{~Hz}]$ \\
Spin-resonance frequency & $1011810 \pm 15[\mathrm{~Hz}]$ \\
Orbit length & $183.4341 \pm 0.0002[\mathrm{~m}]$ \\
Relativistic $\gamma$ factor & $1.9530 \pm 0.0001$ \\
Reconstructed beam momentum & $3146.41 \pm 0.17[\mathrm{MeV} / c]$ \\
\hline \hline
\end{tabular}

Sec. IV C, the measured spin-resonance widths of 80 to $100 \mathrm{~Hz}$ are dominated by the momentum spread. Assuming a Gaussian distribution in the revolution frequency with a FWHM $=40-50 \mathrm{~Hz}$, and neglecting other effects, the width of the spin-resonance distribution requires a momentum spread of $(\delta p / p)_{\mathrm{rms}} \approx 2 \times 10^{-4}$. This upper limit on the beam momentum width corresponds to a smearing of the orbit length of $(\delta s / s)_{\mathrm{rms}} \approx 4 \times 10^{-5}$.

The momentum spread could be checked from the frequency slip factor $\eta$, which was measured at each energy. Using $\delta p / p=1 / \eta \times\left(\delta f_{0} / f_{0}\right)$, this leads, for example, at $p_{\text {nominal }}=3.1625 \mathrm{GeV} / c$ to $(\delta p / p)_{\text {rms }}=1.4 \times 10^{-4}$, which is consistent with the limit obtained from the resonance distribution.

\section{CONCLUSIONS AND OUTLOOK}

In this paper we have shown how to determine the momentum of a deuteron beam in a circular accelerator with high precision using the spin-resonance technique developed at the VEPP accelerator for electron beams. We have studied the depolarization of a polarized deuteron beam at COSY through an induced spin resonance for 13 different beam energies. This was done under standard experimental conditions, i.e., with cavities, in particular the bb cavity, and a thick internal cluster-jet target. The momenta and other beam properties were found by measuring the position of the spin-resonance and revolution frequencies.

It was possible to determine the beam momenta with an accuracy of $\Delta p / p \leq 6 \times 10^{-5}$, i.e., the 13 momenta in the range $3100-3200 \mathrm{MeV} / c$ were measured with precisions of $\approx 170 \mathrm{keV} / c$, a feat never before achieved at COSY. The actual precision was limited by the systematic variations of the orbit length and the characteristics of the Schottky spectrum analyzer. The latter could be improved significantly through the comparison with a calibrated frequency standard.

The orbit length could be extracted from the revolution and spin-resonance frequencies with an accuracy of $\Delta s / s \leq 2 \times 10^{-6}$. Thus for COSY, with a circumference of $183.4 \mathrm{~m}$, the orbit length could be measured with a precision below $0.3 \mathrm{~mm}$. This may allow one to gain a better knowledge of the orbit behavior in COSY.

These results were achieved using a deuteron beam, but there are no in-principle reasons why the depolarization technique should not be applicable to proton beams at COSY with same success.

In summary, the spin-resonance method is a powerful beam diagnostic tool for circular accelerators, synchrotrons, or storage rings without Siberian snakes to investigate and determine beam properties. In our particular case it should eventually allow the mass of the $\eta$ meson to be measured with a precision of $\Delta m_{\eta} \leq 50 \mathrm{keV} / c^{2}$.

\section{ACKNOWLEDGMENTS}

The authors wish to express their thanks to the other members of the COSY machine crew for producing such good experimental conditions and also to the other members of the ANKE collaboration for diverse help in the experiment. The spin-depolarizing studies for deuterons and protons were initiated at COSY by Alan Krisch and other members of the SPIN@COSY collaboration and we have benefited much from their experience. This work was supported in part by the HGF-VIQCD, and JCHP FEE.

[1] C. Amsler et al. (Particle Data Group), Phys. Lett. B 667, 1 (2008).

[2] A. Khoukaz, COSY proposal \#187, 2007, available from www.fz-juelich.de/ikp/anke/en/proposal/ public_proposal_187.pdf.

[3] R. Maier et al., Nucl. Instrum. Methods Phys. Res., Sect. A 390, 1 (1997).

[4] A. Khoukaz et al., Eur. Phys. J. D 5, 275 (1999).

[5] S. Barsov et al., Nucl. Instrum. Methods Phys. Res., Sect. A 462, 364 (2001).

[6] T. Mersmann et al., Phys. Rev. Lett. 98, 242301 (2007).

[7] S. I. Serednyakov et al., Zh. Eksp. Teor. Fiz. 71, 2025 (1976).

[8] Ya. S. Derbenev et al., Part. Accel. 10, 177 (1980).

[9] A. D. Bukin et al., Yad. Fiz. 27, 976 (1978) [Sov. J. Nucl. Phys. 27, 516 (1978)]; A. S. Artamonov et al., Phys. Lett. 118B, 225 (1982); V. M. Aulchenko et al., Phys. Lett. B 573, 63 (2003).

[10] D. P. Barber et al., Phys. Lett. 135B, 498 (1984).

[11] W. W. MacKay et al., Phys. Rev. D 29, 2483 (1984).

[12] L. Arnaudon et al., Phys. Lett. B 284, 431 (1992).

[13] V.S. Morozov et al., Phys. Rev. ST Accel. Beams 7, 024002 (2004).

[14] V.S. Morozov et al., Phys. Rev. ST Accel. Beams 8, 061001 (2005).

[15] H. Stockhorst et al., FZ Jülich IKP Ann. Report, 2007, p. 22; M. Hartmann, Int. J. Mod. Phys. E 18, 465 (2009).

[16] M. Altmeier et al., Eur. Phys. J. A 23, 351 (2005), and references therein.

[17] G. G. Ohlsen, Rep. Prog. Phys. 35, 717 (1972).

[18] L. H. Thomas, Philos. Mag. 3, 1 (1927); V. Bargmann, L. Michel, and V. L. Telegdi, Phys. Rev. Lett. 2, 435 (1959).

[19] Generally the $g$ factor of the deuteron is written in units of the nuclear magneton. If it is required in terms of the deuteron magneton, this has to be calculated from the $g$ factor of the proton and the ratios of the magnetic moment 
and mass of proton and deuteron: $g_{d}=\frac{1}{2} g_{p} \mu_{d} m_{d} / \mu_{p} m_{p}$. The constants required can be found in the NIST compilation [20], but in the evaluation of the uncertainty one has taken into account the fact that the values $\left(\mu_{d} / \mu_{p}, g_{p}\right)$ and $\left(m_{d} / m_{p}, g_{p}\right)$ are correlated. Having done this, we find $G_{d}=-0.1429872725 \pm 0.0000000073$.

[20] NIST-National Institute for Standards and Technology, http://physics.nist.gov/cuu/Constants/index.html.

[21] A. Lehrach et al., AIP Conf. Proc. 675, 153 (2003).

[22] S. Y. Lee, Spin Dynamics and Snakes in Synchrotrons (World Scientific, Singapore, 1997), p. 67.

[23] D. Chiladze et al., Phys. Rev. ST Accel. Beams 9, 050101 (2006).
[24] H. J. Stein et al., Phys. Rev. ST Accel. Beams 11, 052801 (2008).

[25] R. Stassen et al., in Proceedings of the 11th European Particle Accelerator Conference, Genoa, 2008 (EPS-AG, Genoa, Italy, 2008), MOPC125.

[26] P. Goslawski, Diploma thesis, Westfälische-WilhelmsUniversität Münster, Germany, 2009.

[27] D. Boussard, CERN Report No. CERN 87-03:41, 1987.

[28] V. Schwarz, Ph.D. thesis, Rheinische-Friedrich-WilhelmsUniversität Bonn, Germany, 1999.

[29] A. P. Lysenko, A. A. Polunin, and Yu. M. Shatunov, Part. Accel. 18, 215 (1986). 